\title{
Faddeev eigenfunctions for point potentials in two dimensions *
}

\author{
P.G. Grinevich ${ }^{\dagger} \quad$ R.G.Novikov ${ }^{\ddagger}$
}

\begin{abstract}
We present explicit formulas for the Faddeev eigenfunctions and related generalized scattering data for point (delta-type) potentials in two dimensions. In particular, we obtain the first explicit examples of such eigenfunctions with contour singularity in spectral parameter at a fixed real energy.
\end{abstract}

\section{Introduction}

Consider the two-dimensional Schrödinger equation

$$
-\Delta \psi+v(x) \psi=E \psi, \quad x \in \mathbb{R}^{2},
$$

where $v(x)$ is a real-valued sufficiently regular function on $\mathbb{R}^{2}$ with sufficient decay at infinity.

For (11) we consider the classical scattering eigenfunctions $\psi^{+}$specified by

$$
\psi^{+}=e^{i k x}-i \pi \sqrt{2 \pi} e^{-\frac{i \pi}{4}} f\left(k,|k| \frac{x}{|x|}\right) \frac{e^{i|k||x|}}{\sqrt{|k||x|}}+o\left(\frac{1}{\sqrt{|x|}}\right), \quad \text { as } \quad|x| \rightarrow \infty
$$

*The work was supported by the the Russian Federation Government grant No 2010220-01-077. The first author was also supported by by LIA "Physique théorique et matière condensée" ENS - LANDAU and by the program "Fundamental problems of nonlinear dynamics" of the Presidium of RAS.

${ }^{\dagger}$ Landau Institute of Theoretical Physics, Kosygin street 2, 117940 Moscow, Russia; e-mail:pgg@landau.ac.ru.

${ }^{\ddagger}$ CNRS (UMR 7641), Centre de Mathématiques Appliquées, École Polytechnique, 91128, Palaiseau, France; e-mail: novikov@cmap.polytechnique.fr 
$k \in \mathbb{R}^{2}, k^{2}=E>0$, where a priori unknown function $f(k, l), k, l \in \mathbb{R}^{2}$, $k^{2}=l^{2}=E$, arising in (2), is the classical scattering amplitude for (1). In addition, we consider the Faddeev eigenfunctions $\psi$ for (1) (see [7], [13], [9]), specified by

$$
\psi=e^{i k x}(1+o(1)) \text { as }|x| \rightarrow \infty,
$$

$k \in \mathbb{C}^{2}, \operatorname{Im} k \neq 0, k^{2}=E$. The generalized scattering data arise in more precise version of the expansion (3) (see also formulas (71)-(12)). The Faddeev eigenfunctions are quite important for inverse scattering (see, for example, [8], [12, [9]).

In addition, as regards basic results on inverse scattering at fixed energy in two dimensions see [10], [5], [11], [13], [14], 9] and references therein. In addition, as regards potentials, for which direct and inverse scattering at fixed energy in two dimensions is exactly solvable see [9], [15] and references therein.

However, modern monochromatic 2D inverse scattering is well-developed under the assumption, that the Faddeev eigenfunctions has no singularities in spectral parameter at fixed energy in complex domain. Due to [11] this condition is restrictive. In particular, in [11] it was shown, that at a negative energy $E$ above the ground state one can expect contour singularities in the complex plane of spectral parameter $\lambda$, in typical situation. But the theory of generalized analytic functions, used for monochromatic inverse scattering (see equations (17)-(19) on Faddeev eigenfunctions) is not developed for such singularities.

In addition, no example of potential, for which direct and inverse scattering in two dimensions is explicitly solvable for each energy from some non-empty open interval, was given in literature. Besides, no example of potential, for which the Faddeev eigenfunctions at a fixed energy in two dimensions are calculated explicitly and have the aforementioned contour singularities, was given in literature. May be the latter example for zero energy can be extracted from [15] (private communication by I.A. Taimanov).

In the present article we consider equation (1), where $v(x)$ is the 2dimensional analog of the 3-dimensional point potential of Zeldovich [16] and Berezin-Faddeev [4]. Following [4] we will write

$$
v(x)=\varepsilon \delta(x),
$$

but the precise sense of this potential will be specified below (see Section 3), and strictly speaking, $\delta(x)$ is not the standard Dirac delta-function. We show, 
that this potential can be considered as an explicitly solvable model for direct and inverse scattering for (1) at each real energy $E$. In particular, we obtain explicit formulas for related Faddeev eigenfunctions. These eigenfunctions have the aforementioned contour singularities in spectral parameter $\lambda$ if $|E|$ is sufficiently small. We hope, that this example will help to find correct formulation of monochromatic inverse scattering in two dimensions in the presence of spectral contour singularities.

Generalizations of the results of the present article for a sum of several point potentials in $2 \mathrm{D}$ and in $3 \mathrm{D}$ will be given elsewhere.

\section{Some preliminaries}

It is convenient to write

$$
\psi=e^{i k x} \mu
$$

where $\psi$ solves (3) and $\mu$ solves

$$
-\Delta \mu-2 i k \nabla \mu+v(x) \mu=0, \quad k \in \mathbb{C}^{2}, \quad k^{2}=E .
$$

In addition, to relate eigenfunctions and scattering data it is convenient to use the following presentations, used, for example, in [14] for regular potentials:

$$
\begin{aligned}
& \mu^{+}(x, k)=1-\int_{\mathbb{R}^{2}} \frac{e^{i \xi x} F(k,-\xi)}{\xi^{2}+2(k+i 0 k) \xi} d \xi, \quad k \in \mathbb{R}^{2} \backslash 0, \\
& \mu_{ \pm}(x, k)=1-\int_{\mathbb{R}^{2}} \frac{e^{i \xi x} H_{ \pm}(k,-\xi)}{\xi^{2}+2\left(k \pm i 0 k_{\perp}\right) \xi} d \xi, \quad k \in \mathbb{R}^{2} \backslash 0,
\end{aligned}
$$

where $k_{\perp}=\left(-k_{2}, k_{1}\right)$ for $k=\left(k_{1}, k_{2}\right)$,

$$
\mu(x, k)=1-\int_{\mathbb{R}^{2}} \frac{e^{i \xi x} H(k,-\xi)}{\xi^{2}+2 k \xi} d \xi, \quad k \in \mathbb{C}^{2}, \quad \operatorname{Im} k \neq 0,
$$

where $\psi^{+}=e^{i k x} \mu^{+}$are the eigenfunctions, specified by (2), $\psi=e^{i k x} \mu$ are the eigenfunctions, specified by (3) $, \mu_{ \pm}(x, k)=\mu\left(x, k \pm i 0 k_{\perp}\right), k \in \mathbb{R}^{2} \backslash 0$.

The following formulas holds:

$$
f(k, l)=F(k, k-l), \quad k, l \in \mathbb{R}^{2}, \quad k^{2}=l^{2}=E>0,
$$




$$
\begin{gathered}
h_{ \pm}(k, l)=H_{ \pm}(k, k-l), \quad k, l \in \mathbb{R}^{2}, \quad k^{2}=l^{2}=E>0, \\
a(k)=H(k, 0), \quad b(k)=H(k, 2 \operatorname{Re} k), \quad k \in \mathbb{C}^{2} \backslash \mathbb{R}^{2}, \quad k^{2}=E \in \mathbb{R},
\end{gathered}
$$

where $f(k, l)$ is the classical scattering amplitude (22), $h_{ \pm}(k, l), a(k), b(k)$ are Faddeev generalized scattering data.

Let us recall, that the fixed energy surface $\Sigma_{E}=\left\{k \in \mathbb{C}^{2}: k^{2}=E\right\}$ can be parametrized as follows:

$$
\begin{gathered}
\Sigma_{0}=\Sigma_{0}^{+} \cup \Sigma_{0}^{-}, \quad \Sigma_{0}^{ \pm}=\left\{k_{0}^{ \pm}(\lambda)=(\lambda, \pm i \lambda): \lambda \in \mathbb{C}\right\} \\
\Sigma_{E}=\left\{k_{E}(\lambda)=\left(\left(\frac{1}{\lambda}+\lambda\right) \frac{\sqrt{E}}{2},\left(\frac{1}{\lambda}-\lambda\right) \frac{i \sqrt{E}}{2}\right): \lambda \in \mathbb{C}\right\}, \quad E \neq 0 .
\end{gathered}
$$

In addition:

$$
\begin{gathered}
\left|\operatorname{Re} k_{0}^{ \pm}(\lambda)\right|+\left|\operatorname{Im} k_{0}^{ \pm}(\lambda)\right|=2|\lambda|, \\
\left|\operatorname{Re} k_{E}(\lambda)\right|+\left|\operatorname{Im} k_{E}(\lambda)\right|=\left\{\begin{array}{ll}
\sqrt{|E|}|\lambda|, & |\lambda| \geq 1, \\
\sqrt{|E|}|\lambda|^{-1}, & |\lambda|<1,
\end{array} \quad E \neq 0 .\right.
\end{gathered}
$$

Note, that for regular real-valued potentials the following formulas hold (at least outside the singularities of Faddeev functions in spectral parameter):

$$
\begin{gathered}
\frac{\partial}{\partial \bar{\lambda}} \psi\left(x, k_{E}(\lambda)\right)=\frac{\pi \operatorname{sign}(\lambda \bar{\lambda}-1)}{\bar{\lambda}} b\left(k_{E}(\lambda) \overline{\psi\left(x, k_{E}(\lambda)\right)}, \quad E<0 \quad \lambda \in \mathbb{C}, \quad(17)\right. \\
\frac{\partial}{\partial \bar{\lambda}} \psi\left(x, k_{0}^{ \pm}(\lambda)\right)=\frac{\pi}{\bar{\lambda}} b\left(k_{0}^{ \pm}(\lambda) \overline{\psi\left(x, k_{0}^{ \pm}(\lambda)\right)}, \quad \lambda \in \mathbb{C} \backslash 0,\right. \\
\frac{\partial}{\partial \bar{\lambda}} \psi\left(x, k_{E}(\lambda)\right)=\frac{\pi \operatorname{sign}(\lambda \bar{\lambda}-1)}{\bar{\lambda}} b\left(k_{E}(\lambda) \overline{\psi\left(x, k_{E}(\lambda)\right)}, \quad E>0, \lambda \in \mathbb{C},|\lambda| \neq 1,\right.
\end{gathered}
$$

$$
\begin{aligned}
& \psi_{ \pm}\left(x, k_{E}(\lambda)\right)=\psi^{+}\left(x, k_{E}(\lambda)\right)+\pi i \int_{\left|\lambda^{\prime}\right|=1} h_{ \pm}\left(k_{E}(\lambda), k_{E}\left(\lambda^{\prime}\right)\right) \times \\
& \times \chi_{+}\left( \pm i\left(\frac{\lambda}{\lambda^{\prime}}-\frac{\lambda^{\prime}}{\lambda}\right)\right) \psi^{+}\left(x, k_{E}\left(\lambda^{\prime}\right)\right)\left|d \lambda^{\prime}\right|, \quad E>0, \lambda, \lambda^{\prime} \in \mathbb{C},|\lambda|=1,
\end{aligned}
$$




$$
\begin{gathered}
h_{ \pm}\left(k_{E}(\lambda), k_{E}\left(\lambda^{\prime}\right)\right)-\pi i \int_{\left|\lambda^{\prime \prime}\right|=1} h_{ \pm}\left(k_{E}(\lambda), k_{E}\left(\lambda^{\prime \prime}\right)\right) \chi_{+}\left( \pm i\left(\frac{\lambda}{\lambda^{\prime \prime}}-\frac{\lambda^{\prime \prime}}{\lambda}\right)\right) \times \\
\times f\left(k_{E}\left(\lambda^{\prime \prime}\right), k_{E}\left(\lambda^{\prime}\right)\right)\left|d \lambda^{\prime \prime}\right|=f\left(k_{E}(\lambda), k_{E}\left(\lambda^{\prime}\right)\right), \\
E>0, \lambda, \lambda^{\prime}, \lambda^{\prime \prime} \in \mathbb{C},|\lambda|=\left|\lambda^{\prime}\right|=1 \\
\mu\left(x, k_{E}(\lambda)\right) \rightarrow 1 \text { for } \lambda \rightarrow \infty, \quad E \in \mathbb{R}
\end{gathered}
$$

where

$$
\chi_{+}(s)=1 \text { for } s>0, \chi_{+}(s)=0 \text { for } s \leq 0,
$$

and $\mu$ is related with $\psi$ as in (15); see [10], [5], [11], [13], [9] and references therein. In particular, formulas of the type (20), (21) go back to [8], formulas of the type (17)-(19) go back to [1], 3] . In addition, let us recall that formulas (17)-(22) give a basis for monochromatic inverse scattering for regular realvalued potentials in two dimensions.

\section{Main results}

By analogy with [4] we understand the point-like potential $v(x)$ from (44) as a limit for $N \rightarrow+\infty$ of non-local potentials $V_{N}\left(x, x^{\prime}\right)=\varepsilon(N) u_{N}(x) u_{N}\left(x^{\prime}\right)$ where,

$$
\begin{gathered}
\left(V_{N} \mu\right)(x)=\varepsilon(N) \int_{\mathbb{R}^{2}} u_{N}(x) u_{N}\left(x^{\prime}\right) \mu\left(x^{\prime}\right) d x^{\prime}, \\
u_{N}(x)=\left(\frac{1}{2 \pi}\right)^{2} \int_{\mathbb{R}^{2}} \hat{u}_{N}(\xi) e^{i \xi x} d \xi, \quad \hat{u}_{N}(\xi)= \begin{cases}1 & |\xi| \leq N, \\
0 & |\xi|>N,\end{cases}
\end{gathered}
$$

$\varepsilon(N)$ is normalizing constant specified by (29).

For $v=V_{N}$ equation (66) has the following explicit solutions:

$$
\begin{gathered}
\mu_{N}(x, k)=1+\left(\frac{1}{2 \pi}\right)^{2} \int_{\mathbb{R}^{2}} \tilde{\mu}_{N}(\xi, k) e^{i \xi x} d \xi \\
\tilde{\mu}_{N}(\xi, k)=-\frac{\varepsilon(N) u_{N}(0) u_{N}(\xi)}{1+\varepsilon(N)\left(\frac{1}{2 \pi}\right)^{2} \int_{\mathbb{R}^{2}} \frac{u_{N}(-\zeta) u_{N}(\zeta)}{\zeta^{2}+2 k \zeta} d \zeta} \cdot \frac{1}{\xi^{2}+2 k \xi},
\end{gathered}
$$


where $x \in \mathbb{R}^{2}, \xi \in \mathbb{R}^{2}, k \in \mathbb{C}^{2}, \operatorname{Im} k \neq 0$. In addition, equation (6) has the following classical scattering solutions:

$$
\mu_{N}^{+}(x, k)=\mu_{N}(x, k+i 0 k), \quad x \in \mathbb{R}^{2}, \quad k \in \mathbb{R}^{2} \backslash 0,
$$

arising from

$$
\tilde{\mu}_{N}^{+}(\xi, k)=\tilde{\mu}_{N}(\xi, k+i 0 k), \quad \xi \in \mathbb{R}^{2}, \quad k \in \mathbb{R}^{2} \backslash 0 .
$$

Theorem 1 Let

$$
\varepsilon(N)=\frac{\alpha}{1-\frac{\alpha}{2 \pi} \ln (N)}, \quad \alpha \in \mathbb{R}
$$

Then:

1. The limiting eigenfunctions

$$
\psi(x, k)=e^{i k x} \lim _{N \rightarrow+\infty} \mu_{N}(x, k), \quad \psi^{+}(x, k)=e^{i k x} \lim _{N \rightarrow+\infty} \mu_{N}^{+}(x, k)
$$

are well-defined for $x \in \mathbb{R}^{2}$ and $k$ as indicated for (2), (3).

2. The following formulas hold:

$$
\begin{gathered}
\psi(x, k)=e^{i k x}\left[1+\frac{\alpha}{1-\frac{\alpha}{2 \pi} \ln (|\operatorname{Re} k|+|\operatorname{Im} k|)} \cdot g(x, k)\right] \\
g(x, k)=-\left(\frac{1}{2 \pi}\right)^{2} \int_{\mathbb{R}^{2}} \frac{e^{i \xi x}}{\xi^{2}+2 k \xi} d \xi, \quad k \in \mathbb{C}^{2}, \quad \operatorname{Im} k \neq 0, \quad k^{2}=E \in \mathbb{R} \\
\psi^{+}(x, k)=e^{i k x}\left[1+\frac{\alpha}{1+\frac{\alpha}{4 \pi}(\pi i-2 \ln |k|)} \cdot g^{+}(x, k)\right] \\
g^{+}(x, k)=-\left(\frac{1}{2 \pi}\right)^{2} \int_{\mathbb{R}^{2}} \frac{e^{i \xi x}}{\xi^{2}+2(k+i 0 k) \xi} d \xi, \quad k \in \mathbb{R}^{2}, \quad k \neq 0 \\
\psi_{ \pm}(x, k)=\psi\left(x, k \pm i 0 k_{\perp}\right)=e^{i k x}\left[1+\frac{\alpha}{1-\frac{\alpha}{2 \pi} \ln |k|} \cdot g_{ \pm}(x, k)\right] \\
g_{ \pm}(x, k)=-\left(\frac{1}{2 \pi}\right)^{2} \int_{\mathbb{R}^{2}} \frac{e^{i \xi x}}{\xi^{2}+2\left(k \pm i 0 k_{\perp}\right) \xi} d \xi, \quad k \in \mathbb{R}^{2}, \quad k \neq 0
\end{gathered}
$$


3. The scattering data for the limiting potential $v=\lim _{N \rightarrow+\infty} V_{N}$, associated with the limiting eigenfunctions $\psi^{+}(x, k), \psi_{ \pm}(x, k), \psi(x, k)$ are given by:

$$
\begin{gathered}
f(k, l)=\left(\frac{1}{2 \pi}\right)^{2} \frac{\alpha}{1+\frac{\alpha}{4 \pi}(\pi i-2 \ln |k|)} \\
h_{ \pm}(k, l)=\left(\frac{1}{2 \pi}\right)^{2} \frac{\alpha}{1-\frac{\alpha}{2 \pi} \ln (|k|)}
\end{gathered}
$$

where $k, l \in \mathbb{R}^{2}, k^{2}=l^{2}=E>0$;

$$
a(k)=b(k)=\left(\frac{1}{2 \pi}\right)^{2} \frac{\alpha}{1-\frac{\alpha}{2 \pi} \ln (|\operatorname{Re} k|+|\operatorname{Im} k|)},
$$

where $k \in \mathbb{C}^{2}, \operatorname{Im} k \neq 0, k^{2}=E \in \mathbb{R}$.

Remark 1 Relations between the absolute value of the scattering amplitude $f$ and its phase for a two-dimensional point-like scatterer was given earlier in [6], see also [2] for further development. To our knowledge no exact formulas for the Faddeev eigenfunctions $\psi$ and related scattering data $a(k), b(k)$ associated with $2 D$ point potentials were given in the literature.

Proposition 1 Formulas (17)-(22) are fulfilled for functions $\psi=e^{i k x} \mu$, $\psi^{+}=e^{i k x} \mu^{+}, \psi_{ \pm}=e^{i k x} \mu_{ \pm}, a, b, f, h_{ \pm}$of Theorem 1, at least for $x \neq 0$.

In addition, quite interesting properties of these functions for $\alpha \neq 0$ can be summarized as the following statement:

Statement 1 Let $\alpha \neq 0, E_{1}=-\exp \left(\frac{4 \pi}{\alpha}\right), x \neq 0$. Then:

1. If $E<E_{1}$, then the functions $\mu\left(x, k_{E}(\lambda)\right), a\left(k_{E}(\lambda)\right), b\left(k_{E}(\lambda)\right)$ are continuous in $\lambda \in(\mathbb{C} \cup \infty)$.

2. If $E=E_{1}$, then the functions $\mu\left(x, k_{E}(\lambda)\right), a\left(k_{E}(\lambda)\right), b\left(k_{E}(\lambda)\right)$ are singular in $\lambda$ on the contour

$$
C_{E_{1}, \alpha}=T=\{\lambda \in \mathbb{C}:|\lambda|=1\}
$$

and are continuous in $\lambda \in(\mathbb{C} \cup \infty) \backslash T$. In addition, this energy level $E_{1}$ is discrete eigenvalue with eigenfunction

$$
\psi_{1}(x)=-\left(\frac{1}{2 \pi}\right)^{2} \int_{\mathbb{R}^{2}} \frac{e^{i \xi x}}{\xi^{2}-E_{1}} d \xi .
$$


3. If $E_{1}<E<0$, then the functions $\mu\left(x, k_{E}(\lambda)\right)$, a $\left(k_{E}(\lambda)\right), b\left(k_{E}(\lambda)\right)$ have simple singularities in $\lambda$ on the contours

$$
C_{E, \alpha}=\left\{\lambda \in \mathbb{C}:|\lambda|=\sqrt{\left|E / E_{1}\right|}\right\} \cup\left\{\lambda \in \mathbb{C}:|\lambda|=\sqrt{\left|E_{1} / E\right|}\right\}
$$

and are continuous in $\lambda \in(\mathbb{C} \cup \infty) \backslash C_{E, \alpha}$.

4. At zero energy $E=0$ the functions $\mu\left(x, k_{0}^{ \pm}(\lambda)\right), a\left(k_{0}^{ \pm}(\lambda)\right), b\left(k_{0}^{ \pm}(\lambda)\right)$ have simple singularities in $\lambda$ on the contour

$$
C_{0, \alpha}=\left\{\lambda \in \mathbb{C}:|\lambda|=\frac{1}{2} \sqrt{\left|E_{1}\right|}\right\}
$$

and are continuous in $\lambda \in \mathbb{C} \backslash\left(C_{0, \alpha} \cup 0\right)$ on both components $\Sigma_{0}^{+}, \Sigma_{0}^{-}$.

5. If $0<E<\left|E_{1}\right|$, then the functions $\mu\left(x, k_{E}(\lambda)\right)$, $a\left(k_{E}(\lambda)\right), b\left(k_{E}(\lambda)\right)$ have simple singularities in $\lambda$ on the contours

$$
C_{E, \alpha}=\left\{\lambda \in \mathbb{C}:|\lambda|=\sqrt{\left|E / E_{1}\right|}\right\} \cup\left\{\lambda \in \mathbb{C}:|\lambda|=\sqrt{\left|E_{1} / E\right|}\right\}
$$

and are continuous in $\lambda \in(\mathbb{C} \cup \infty) \backslash\left(C_{E, \alpha} \cup T\right)$.

In addition, $\mu\left(x, k_{E}(\lambda(1 \mp 0))=\mu_{ \pm}\left(x, k_{E}(\lambda)\right)\right.$ for $\lambda \in T$, where $\mu_{ \pm}$are continuous in $\lambda \in T$ (whereas $a\left(k_{E}(\lambda)\right), b\left(k_{E}(\lambda)\right)$ are continuous in a neighborhood of $T$ ).

6. If $E=\left|E_{1}\right|$, then the functions $\mu\left(x, k_{E}(\lambda)\right), a\left(k_{E}(\lambda)\right), b\left(k_{E}(\lambda)\right)$ are singular in $\lambda$ on the contour

$$
T=\{\lambda \in \mathbb{C}:|\lambda|=1\}
$$

and are continuous in $\lambda \in(\mathbb{C} \cup \infty) \backslash T$. In addition, $\mu_{ \pm}, h_{ \pm}$are not well-defined in this case; for this energy we have real exceptional points for the Faddeev eigenfunctions.

7. If $E>\left|E_{1}\right|$, then the functions $\mu\left(x, k_{E}(\lambda)\right), a\left(k_{E}(\lambda)\right), b\left(k_{E}(\lambda)\right)$ are continuous in $\lambda \in(\mathbb{C} \cup \infty) \backslash T$.

In addition, $\mu\left(x, k_{E}(\lambda(1 \mp 0))=\mu_{ \pm}\left(x, k_{E}(\lambda)\right)\right.$ for $\lambda \in T$, where $\mu_{ \pm}$are continuous in $\lambda \in T$ (whereas $a\left(k_{E}(\lambda)\right), b\left(k_{E}(\lambda)\right)$ are continuous in a neighborhood of $T$ ). 


\section{Sketch of proofs}

The proof of items 1 and 2 of Theorem 1 follows from direct calculations using the following formulas:

$$
\begin{gathered}
\int_{\xi \in \mathbb{R}^{2},|\xi| \leq N} \frac{e^{i \xi x}}{\xi^{2}+2 k \xi-i 0} d \xi=\int_{\xi \in \mathbb{R}^{2},|\xi| \leq N} \frac{e^{i \xi x}}{\xi^{2}+2|k| \xi_{1}-i 0} d \xi=2 \pi \ln N+ \\
+\pi^{2} i-4 \int_{0}^{\pi / 2} \ln (2 s \cos \phi) d \phi+O\left(N^{-1}\right), \quad k \in \mathbb{R}^{2} \backslash 0, \quad N \rightarrow+\infty, \quad \\
\int_{\xi \in \mathbb{R}^{2},|\xi| \leq N} \frac{e^{i \xi x}}{\xi^{2}+2 k \xi} d \xi=\int_{\xi \in \mathbb{R}^{2},|\xi| \leq N} \frac{e^{i \xi x}}{\xi^{2}+2|\operatorname{Re} k| \xi_{1}+2 i|\operatorname{Im} k| \xi_{2}} d \xi= \\
=2 \pi \ln N-4 \int_{0}^{\pi / 2} \ln \left(2 \sqrt{|\operatorname{Re} k|^{2} \cos ^{2} \phi+|\operatorname{Im} k|^{2} \sin ^{2} \phi}\right) d \phi+O\left(N^{-1}\right), \\
k \in \mathbb{C}^{2} \backslash \mathbb{R}^{2}, \quad k^{2}=E \in \mathbb{R}, \quad N \rightarrow+\infty
\end{gathered}
$$

where $\xi=\left(\xi_{1}, \xi_{2}\right)$;

$$
\int_{0}^{\pi / 2} \ln (\cos \phi) d \phi=-\frac{\pi}{2} \ln 2
$$

$$
\int_{0}^{\pi / 2} \ln \left(a^{2} \cos ^{2} \phi+b^{2} \sin ^{2} \phi\right) d \phi=\pi \ln \frac{|a|+|b|}{2}, \quad a, b \in \mathbb{R} \backslash 0 .
$$

To prove item 3 of Theorem 1 we use, in addition, formulas (7)-(12).

Proposition 1 and Statement 1 can be proved by direct calculations proceeding from Theorem 1 and the well-known properties of the Faddeev Green function $G=e^{i k x} g$ (see, for example, [5], [13]).

\section{References}

[1] M.J. Ablowitz, D. Bar Jaakov, A.S. Fokas, On the inverse scattering of the time-dependent Schrödinger equation and the associated 
Kadomtsev-Petviashvili equation, Stud. in applied math. 69:2 (1983), $135-143$.

[2] N.P. Badalyan, V.A. Burov, S.A.Morozov, O.D. Rumyantseva, Scattering by acoustic boundary scattering with small wave sizes and their reconstruction. Akusticheskiน Zhurnal, 55:1 (2009), 3-10 (Rusian); English translation: Acoustical Physics 55:1 (2009), 1-7.

[3] R.Beals, R.R.Coifman, Multidimensional inverse scattering and nonlinear partial differential equations, Proc. of Symposia in Pure Mathematics 43 (1985) 45-70.

[4] F.A. Berezin and L.D. Faddeev, Remark on Schrödinger equation with singular potential, Dokl. Akad. Nauk SSSR 137 (1961), 1011-1014 (Rusian); English translation: Soviet Mathematics 2 (1961), 372-375.

[5] M. Boiti, J. Leon, M. Manna, F. Pempinelli, On a spectral transform of a KDV-like equation related to the Schrdinger operator in the plane, Inverse Problems 3, 25-36 (1987).

[6] V.A. Burov, S.A.Morozov, Relationship between the amplitude and phase of a signal scattering by a point-like acoustic inhomogeneity, Akusticheskiu Zhurnal, 47:6 (2001), 751-756 (Rusian); English translation: Acoustical Physics, 47:6 (2001), 659-664.

[7] L.D. Faddeev, Growing solutions of the Schrödibger equation, Dokl. Akad. Nauk SSSR 165 (1965), 514-517 (Rusian); English translation: Soviet Phys. Dokl. 10 (1965), 1033-1035.

[8] L.D. Faddeev, Inverse problem of quantum scattering theory. II. Itogi Nauki i Tekhniki. Sovr. Probl. Math. VINITI, 3 (1974), 93-180 (Rusian); English translation: Journal of Soviet Mathematics, 5:3 (1976), $334-396$.

[9] P.G. Grinevich, The scattering transform for the two-dimensional Schrödinger operator with a potential that decreases at infinity at fixed nonzero energy, Uspekhi Mat. Nauk 55:6(336) (2000), 3-70 (Russian); English translation: Russian Math. Surveys 55:6 (2000), 1015-1083.

[10] P.G. Grinevich, S.V. Manakov, Inverse scattering problem for the twodimensional Schrodinger operator, $\bar{\partial}$ - method and nonlinear equations, 
Funct. Anal. i ego Pril., 20:1 (1986), 14-24 (Russian); English translation: Funct. Anal. Appl., 20, (1986), 94-103.

[11] P.G. Grinevich, S.P. Novikov, Two-dimensional 'inverse scattering problem' for negative energies and generalized-analytic functions. 1. Energies below the ground state, Funct. Anal. i ego Pril.22:1 (1988), 23-33 (Rusian); English translation: Funct. Anal. Appl. 22 (1988), 1927.

[12] G.M. Henkin, R.G. Novikov, The $\bar{\partial}$-equation in the multidimensional inverse scattering problem, Uspekhi Mat. Nauk 42:3(255) (1987), 93152 (Russian); English translation: Russian Math. Surveys 42:3 (1987) 109-180.

[13] R.G. Novikov, The inverse scattering problem on a fixed energy level for the two-dimensional Schrödinger operator. J. Funct. Anal. 103:2 (1992), 409-463.

[14] R.G. Novikov, Approximate solution of the inverse problem of quantum scattering theory with fixed energy in dimension 2, Tr. Mat. Inst. Steklova 225:2 (1999), Solitony Geom. Topol. na Perekrest., 301-318 (Russian); English translation: Proc. Steklov Inst. Math. 225:2 (1999), $285-302$

[15] I.A. Taimanov, S.P. Tsarev, On the Moutard transformation and its applications to spectral theory and soliton equations. Sovrem. Mat. Fundam. Napravl. 35 (2010), 101-117, (Russian).

[16] Ya.B. Zel'dovich, Scattering by a singular potential in perturbation theory and in the momentum representation. Z. Eksper. Teoret. Fiz. 38 (1960), 819-824 (Russian. English summary); English translation: Soviet Physics. JETP 11 (1960), 594-597. 